\title{
On the need to specify and verify standard functions
}

\author{
N. V. Shilov
}

\begin{abstract}
The problem of validation of standard mathematical functions and libraries is well-recognized by industrial and academic professional community but still is poorly understood by freshmen and inexperienced developers. The paper gives and discusses two examples (from the author's pedagogical experience) when formal specification and verification of standard functions do help and are needed.
\end{abstract}

Keywords: mathematical functions, standard libraries, formal specification, formal program verification.

\section{1. $\pi$ is 4}

\subsection{What is $\pi$ ?}

How I want a drink, alcoholic of course, after the heavy lectures involving quantum mechanics. James Jeans (1877-1946), British Scientist [17]

The mathematical irrational number $\pi$ is the ratio of a circle's circumference to its diameter $D$; it is also a well-known mathematical fact that the area of the circle is $\left(\pi \times D^{2}\right) / 4$, i.e. it is $\pi / 4$ of the area of the square built on the circle's diameter. This observation leads to the Monte Carlo method $^{1}$ for computing an approximation of $\pi$ as follows (Figure 1): draw a segment of a circle in the first quadrant and the square around it, then randomly place dots in the square; the ratio of the number of dots inside the circle to the total number of dots should be approximately equal to $\pi / 4$. For example, the series of trials depicted in the figure gives $\pi / 4 \approx \frac{8}{11}$, i.e. $\pi \approx 2 .(90)$.

Of course, the above approximation of $\pi$ as 2.(90) is not a good one. Fortunately, almost everyone remembers a much better approximation 3.14 for $\pi$. Moreover there are many ways to memorize more digits than 3 as above. One way is to memorize a story in which the word lengths represent the digits of $\pi$ : the first word has 3 letters, the second has 1 letter, the third has 4 letters, and so on; in particular, the epigraph of this section is an example of a story to memorize 15 digits of the number.

\footnotetext{
${ }^{1}$ The Monte Carlo method is not adaptive and is very slow compared to other methods to compute $\pi$.
} 


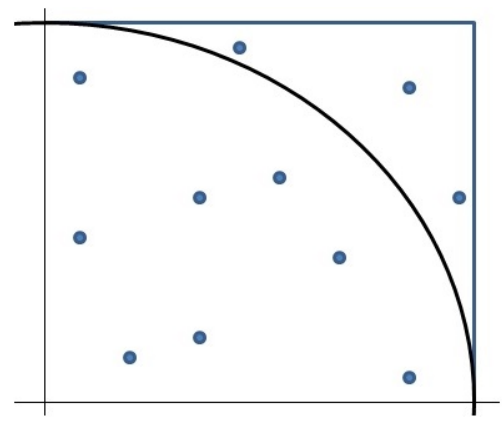

Figure 1. The Monte Carlo method to compute $\pi$

Some computer languages have a standard function to compute $\pi$ approximations. For example, the official site support.office.com specifies a standard PI function and how to use it as follows [18]:

\section{PI function}

This article describes the formula syntax and usage of the PI function in Microsoft Excel.

\section{Description}

Returns the number 3.14159265358979, the mathematical constant pi, accurate to 15 digits.

\section{Syntax}

$\mathrm{PI}()$

The PI function syntax has no arguments

\section{2. $\pi$ by Monte Carlo}

An error becomes an error when born as truth. Stanisław Jerzy Lec (1909-1966), Polish poet and aphorist [19]

The C-program depicted in Figure 2 implements the above Monte Carlo method to compute an approximation for $\pi$. It prescribes to exercise 10 series of 1,000,000 trials each. This code was developed by a Computer Science instructor to teach first-year students C-loops by an example of a very intuitive algorithm. There were 25 students in the class that used either Code::Blocks 12.11 or Eclipse Kepler IDEs for $\mathrm{C} / \mathrm{C}++$ with MinGW environment. Let us refer to this program as PiMC ( $\pi$-Monte Carlo) in the sequel.

Imagine the confusion of the instructor when each of 25 students in the class got 10 times the value 4.000000 as an approximation for $\pi$ ! But it was not the last shock for the instructor this day: a Mathematician that came 


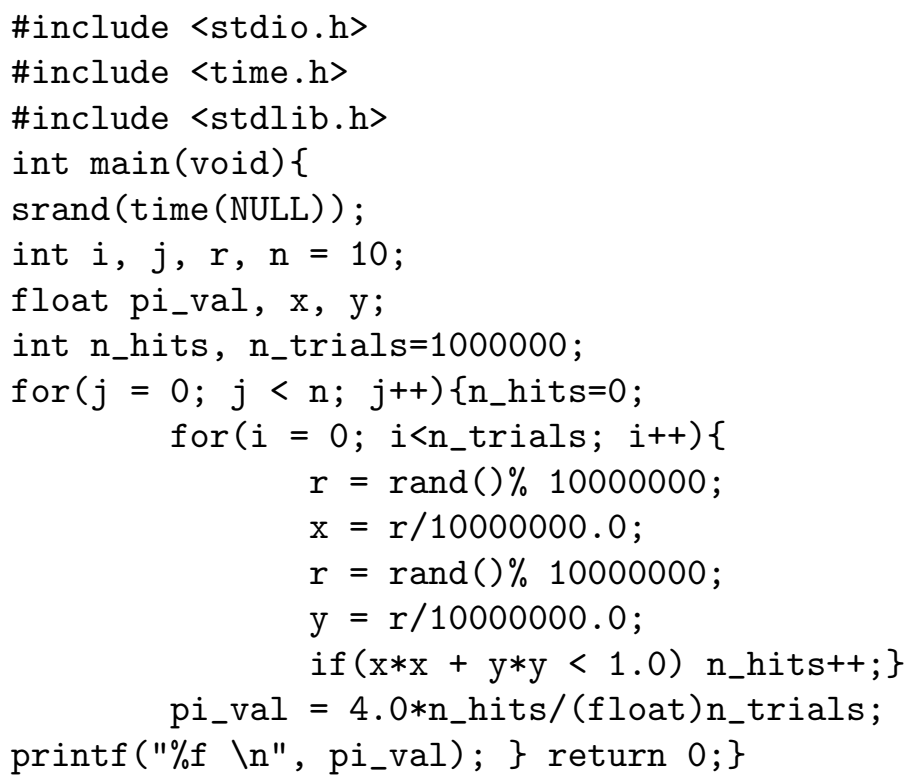

Figure 2. The C-program PiMC to compute $\pi$ approximations

to run the next class had proved that $\pi$ is really 4 . Look at Figure 3 that presents the first three in a sequence of figures circumscribing a circle with a diameter $D$ : every next figure results from the previous one by "cutting corners". The sequence converges to the circle; hence its perimeter converges to $\pi \times D$. But perimeters of all figures in the sequence is a constant $4 D$. Hence $\pi=4$.

So we can summarize: a very intuitive Monte Carlo computational experiment repeated independently 25 times and an obvious proof lead us to a paradoxial conclusion that $\pi$ is 4 .
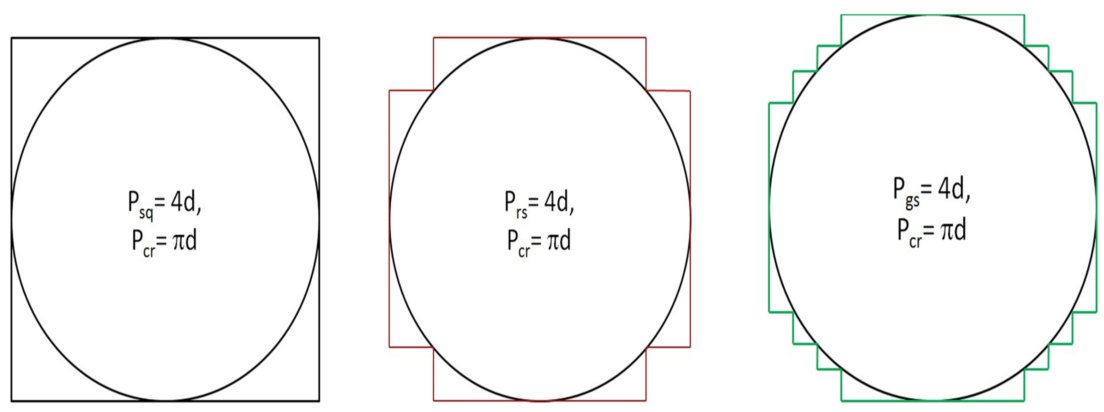

Figure 3. The first three figures of a series converging to a circle 


\subsection{Formal methods as a rescue}

First let us rule out a mathematical "proof" that $\pi=4$ : the mathematical arguments presented here do not prove that $\pi=4$ but demonstrate instead that convergence in the metrics $\mathbf{L}_{\infty}$ does not imply convergence in the metrics $\mathbf{L}_{2}$ [9]: the sequence converges to the circle in the metrics $\mathbf{L}_{\infty}$, the perimeters of all figures are $4 D$, but the sequence does not converge to the circle in the metrics $\mathbf{L}_{2}$ and the circumference of the circle is $\pi \times D \approx 3.14 D$.

Next let us try to figure out what is wrong with the computer program PiMC with the aid of Formal Methods [5]; in particular, let us try to specify the program in the classical Hoare style by pre- and post-conditions [2].

The pre-condition may be TRUE since the program has no input. The post-condition may be pi_val==4.0 since we know from the program exercise the final value of the variable. Due to the exercise, we may formulate the following hypothesis

$$
\models \text { [TRUE] PiMC [pi_val==4.0], }
$$

i.e. the total correctness assertion [TRUE] PiMC [pi_val==4.0] is valid.

If we try to apply the classical verification methods [2] to generate verification conditions and prove the above assertion, we come to a problem of formal semantics of the function rand() in the assignment

$$
r=r a n d() \% 10000000 \text {; }
$$

that has two instances in the program. The standard rule to generate a verification condition for an assignment is

$$
\frac{\phi(x) \rightarrow \psi(t)}{[\phi(x)] x=t[\psi(x)]}
$$

for function rand() it leads to the following rule:

$$
\frac{\phi(x) \rightarrow \psi(\operatorname{rand}())}{[\phi(x)] \mathrm{x}=\operatorname{rand}()[\psi(x)]} .
$$

Unfortunately, we do not know enough about the properties of this function to prove any non-trivial verification condition! In particular, from an intuitive very informal understanding of a random-value generator, rand() should generate with equal probability all values from some range ${ }^{2}$ Range; it implies that the premise $\phi(x) \rightarrow \psi(\operatorname{rand}())$ in the rule for $\operatorname{rand}()$ is equivalent to the formula $\phi(x) \rightarrow \forall x \in$ Range. $\psi(x)$. This verification condition generation rule looks very special (not to say suspicious). For this

\footnotetext{
${ }^{2}$ For the C-language it is known (please refer to the next subsection 1.4) that this Range is an integer interval [0..RAND_MAX].
} 
reason, the Hoare-style verification for probabilistic programs has got little attention in the past [4] and is an actual research topic [16].

Nevertheless, after the above discussion, one may conclude that the cause of wrong $\pi$ approximation by the program PiMC is the use of the assignments (2) and (maybe) the use of the standard function rand(), its poor specification in the language standard and no verification in MinGW.

\subsection{How to fix it}

Remark. This subsection is due to reviewer's comments for the initial version of the paper. The author is very much obliged to the anonymous reviewer and would like to thank him/her for a very valuable addendum.

The C-language reference portal at en.cppreference.com/w/c provides the following loose specification for the function rand () [20]:

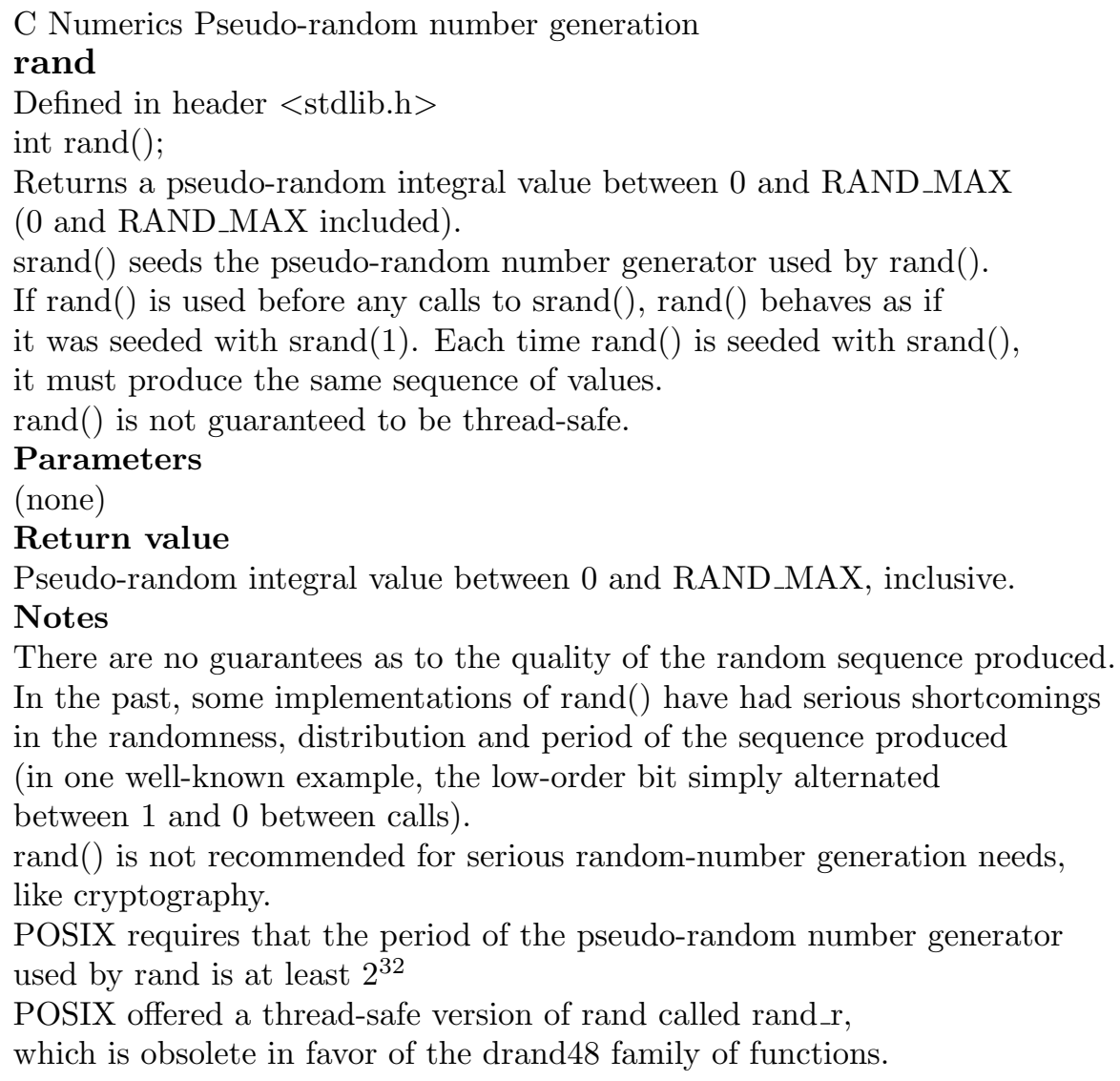

Of course, this specification is neither formal nor accurate and, hence, can not help us to prove every formal property of every program that uses the 
function rand(). Nevertheless, this specification contains enough information to detect what is wrong with the assignment (2) in the program PiMC.

Recall that the function rand() returns an integer in the range from 0 to RAND_MAX inclusively. In many conventional implementations of the C language (including MinGW), RAND_MAX is $2^{15}-1=32767<1000000$. It implies that assignment (2) is simply equivalent to the assignment

$$
r=\operatorname{rand}() \text {; }
$$

Since the value of RAND_MAX in our experiment is just 32767, the normalizing assignments

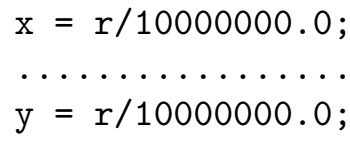

result with the values of $\mathrm{x}$ and $\mathrm{y}$ lying in the range [0,0.032767]; hence, the condition $\mathrm{x} * \mathrm{x}+\mathrm{y} * \mathrm{y}<1.0$ in the $i f$-operator after these assignments is always true and the program PiMC always increments the value of the variable n_hits that counts the number of "randomly" dropped points that have fallen inside the segment of the circle. It implies that after termination of the internal loop for ( $i=0$; $i<n_{-}$trials; $\left.i++\right)$, the values of the variables $n \_$hits and $n \_t r i a l s$ are always equal, and consequently the final value of pi_val is always exactly 4 . We can consider the above discussion as an informal proof for following statement:

$$
\models[\operatorname{rand}() \in[0 \ldots \text { RAND_MAX] \& RAND_MAX==32767] PiMC [pi_val==4.0] . }
$$

The above consideration and discussion lead to the idea how to fix the program PiMC. The body of the internal for-loop should be replaced, for instance, by the following code:

$$
\begin{aligned}
& \mathrm{x}=\operatorname{rand}() /(\text { float }) \text { RAND_MAX; } \\
& \mathrm{y}=\operatorname{rand}() /(\text { l loat }) \text { RAND_MAX; } \\
& \text { if }(\mathrm{x} * \mathrm{x}+\mathrm{y} * \mathrm{y}<1.0) \text { n_hits }++;
\end{aligned}
$$

Let us denote the fixed code by FixPi. Then one can exercise the program and get rather reasonable approximations for the irrational number $\pi$. For example, the anonymous reviewer has got the values $3.140932,3.141289, \ldots$ 3.141315 in a row. The mean value is 3.141353 . Due to the exercise, we may formulate a new hypothesis:

$$
\models \text { [RAND_SPEC \& RAND_MAX==32767] FixMC [3.140<=pi_val<=3.142], }
$$

where RANS_SPEC stands for a formal specification of rand(). But we have to say that we still do not know how a loose specification of rand() from the C-language reference portal can help us to prove or refute the above total correctness assertion. 


\section{What is SQRT?}

\subsection{Solving quadratic equations}

A very popular (but vulgar for professional education) approach to teach standard input/output, floating point data type, sequencing and branching control flow is to program the solving of quadratic equations. (Please check $[7,21,22]$, for instance.) In Figure 4, one can find a variant of a vulgar solver for quadratic equations in the form $a x^{2}+b x+c=0$. We call this solver "vulgar" because none of the conventional computers can solve (in the purely mathematical sense, i.e. find a root) the simple equation $x^{2}-2=0$ (i.e. to compute $\sqrt{2}$ ) due to the irrational nature of the root but the finite size of all numeric data types in every implementation of the $\mathrm{C}$ language.

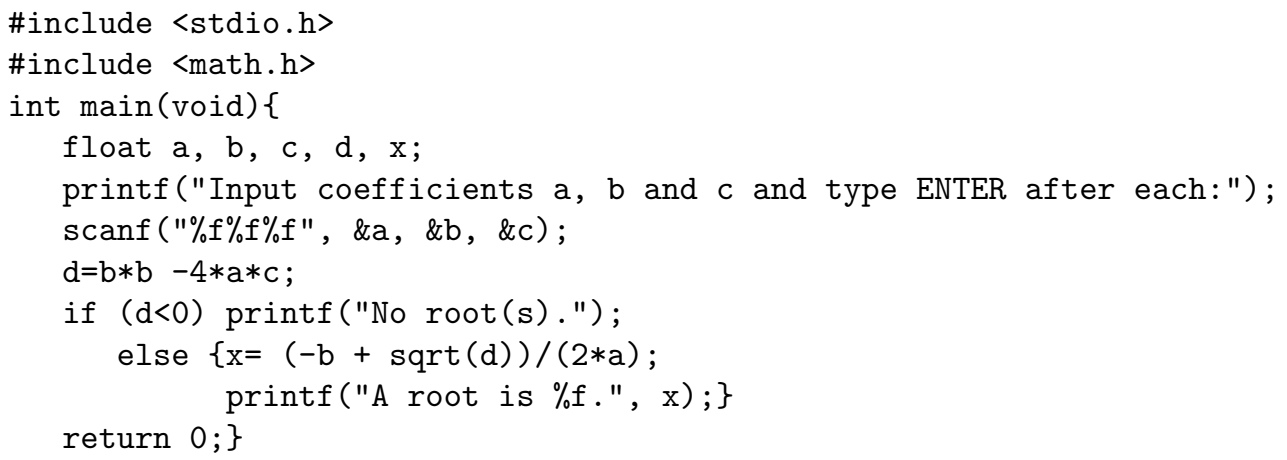

Figure 4. A vulgar solver for quadratic equations

To clarify an ambiguity with the conventional computer ability to solve quadratic equations, let us check what is said at the $\mathrm{C}$ reference portal at en.cppreference.com/w/c regarding the "square root function" sqrt [23]:

C Numerics Common mathematical functions

sqrt, sqrtf, sqrtl

Defined in header $<$ math.h $>$

float sqrtf( float arg ); (1) (since C99)

double sqrt( double arg ); (2)

long double sqrtl( long double arg ); (3) (since C99)

Defined in header $<$ tgmath.h $>$

$\sharp$ define sqrt( arg ) (4) (since C99)

1-3) Computes square root of arg.

4) Type-generic macro: If arg has type long double, sqrtl is called.

Otherwise, if arg has integer type or the type double, sqrt is called.

Otherwise, sqrtf is called. If arg is complex or imaginary,

then the macro invokes the corresponding complex function (csqrtf, csqrt, csqrtl). 


\section{Parameters}

arg - floating point value

Return value

If no errors occur, square root of $\arg (\sqrt{a r g})$, is returned.

If a domain error occurs, an implementation-defined value is returned

(NaN where supported).

If a range error occurs due to underflow, the correct result (after rounding)

is returned.

\section{Error handling}

Errors are reported as specified in math_errhandling.

Domain error occurs if arg is less than zero.

If the implementation supports IEEE floating-point arithmetic (IEC 60559),

- If the argument is less than $-0, \mathrm{FE}$ INVALID is raised and $\mathrm{NaN}$ is returned.

- If the argument is $+\infty$ or \pm 0 , it is returned, unmodified.

- If the argument is $\mathrm{NaN}, \mathrm{NaN}$ is returned

\section{Notes}

sqrt is required by the IEEE standard be exact.

The only other operations required to be exact are the arithmetic operators and the function fma. After rounding to the return type

(using default rounding mode),

the result of sqrt is indistinguishable from the infinitely precise result.

In other words, the error is less than 0.5 ulp.

Other functions, including pow, are not so constrained.

One can see an ambiguity in the specification. According to the above citation, the specification first says that sqrt (2) must be $\sqrt{2}$, but then (in the Notes) that the error of sqrt (2) must be less than 0.5 of ulp $^{3}$ because, by the IEEE standard, the function is required to be exact. Of course, we have to rule out the first option (that sqrt () is $\sqrt{ }$ ) and examine in detail the second one (that sqrt () computes $\sqrt{ }$ with an error less than 0.5 ulp).

The standards mentioned in the specification are IEEE 754-2008 Standard for Floating-Point Arithmetic and the international standard ISO/IEC 60559:2011 [24] (that is identical to IEEE 754-2008). Section 9 of the standard recommends fifty operations that language standards should define (but all these operations are optional, not required in order to conform the standard). But some operations (including sqrt () as a special case of the function ( ) $)^{1 / n}$ for $n=2$ ), if implemented, must round correctly (i.e. with an error less than 0.5ulp).

Another very critical problem with the specification and ISO/IEC/IEEE standards above is the absence (in the specification and standards) of a description of any validation procedure to check/prove that an implementation conforms to the specification/standard.

\footnotetext{
${ }^{3}$ The unit in the least precision (that is type and platform dependable).
} 


\subsection{An alternative for sqrt}

Instead of requiring that sqrt compute the exact irrational square roots for type- and/or platform-dependent approximate values of square roots, it makes sense to specify in the language another "standard" generic function (say $\operatorname{SQR}($,$) ) for a generic numeric type (that is the union of all numeric$ types) with a return value and two parameters of this generic numeric type, where the first parameter is for passing the argument value $Y \geq 0$ and the second is for passing the accuracy value $E>0$; the function is for computing $\sqrt{Y}$ with the accuracy $E$. Let us use the following notation for the function SQR: $S Q R$ is a mathematical function of two real arguments that is computed by SQR, i.e. for every non-negative real number $Y \geq 0$ (that is representable in the generic numeric type) and positive real number $E>0$ (also representable in this type), $\operatorname{SQR}(Y, E)$ returns $S Q R(Y, E)$. The properties of this function $S Q R$ can be formally specified by any (or both) of the following two clauses:

- if $Y \geq 0$ and $E>0$, then $S Q R(Y, E)$ differs from $\sqrt{Y}$ by no more than $E$, i.e. $|S Q R(Y)-\sqrt{Y}| \leq E$;

- if $Y \geq 0$ and $E>0$, then $(S Q R(Y, E))^{2}$ differs from $Y$ by no more than $E$, i.e. $\left|(S Q R(Y))^{2}-Y\right| \leq E$.

It makes sense to fix the first formal specification for better compatibility with the exact standard function, since in this case we can define the standard function sqrt via SQR as follows:

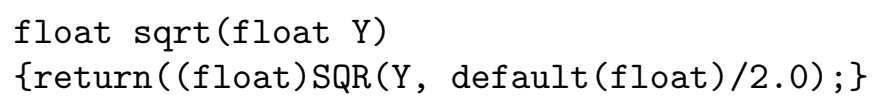

where default is another new type-related feature (similar to sizeof) that returns the value of the unit in the least precision for a numeric type.

One may select any reasonable and feasible computation method to approximate $\sqrt{ }$. For example, it can be a very intuitive Newton-Raphson Method [8]: first guess an initial approximation for the root; then compute the arithmetic mean between the guess and the number (the square root of which you want to obtain) divided by the initial guess; let this mean be a new guess for another go-around while the difference between the next and the previous guesses is bigger than the half of the accuracy. (Please refer to Figure 5 for a sample implementation of the function for the data type float.)

Both floating-point functions in Figure 5 are easy to specify ${ }^{4}$ formally in a Hoare style:

\footnotetext{
${ }^{4}$ Note that the specification is incomplete since it does not specify the exceptional situations (e.g. $Y<0$ and/or $E \leq 0$ ).
} 


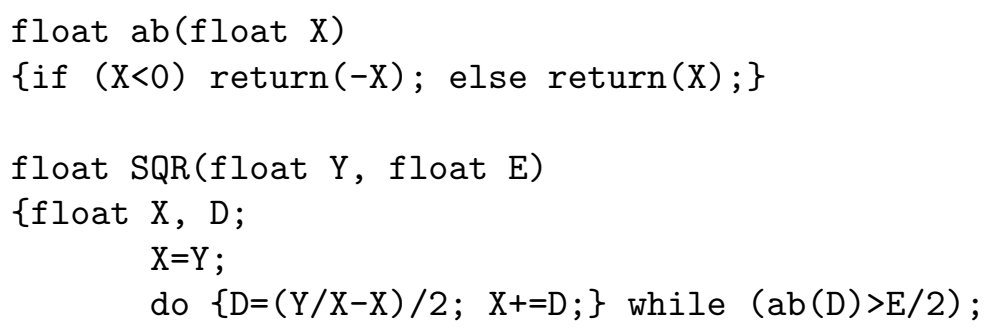

Figure 5. A floating-point function to compute a square root approximation

$[X:$ float $] \mathrm{ab}(X)$ [returned value $=|X|]$, $[Y \geq 0$ and $E>0$ are floats] $\operatorname{SQR}(Y, E)[\mid$ returned value $-\sqrt{Y} \mid \leq E]$.

For a generic function and the generic numeric type, the specification should be modified:

[ TYPE $i$ s a numeric type, $Y \geq 0$ : TYPE and $E>0$ : TYPE]

$$
\operatorname{SQR}(Y, E)[\mid \text { returned value }-\sqrt{Y} \mid \leq E] \text {. }
$$

If these specifications are proved, then SQR may be a good alternative to the standard function sqrt. Unfortunately, it is not easy to prove these specifications automatically and formally because of several reasons. The major one is an axiomatization of the computer floating-point arithmetic $[1,12,25]$. Even a manual pen-and-paper verification of the algorithm $S Q R$ (assuming precise arithmetic for real numbers) is not a trivial exercise which we will solve in the next subsection.

\subsection{Toward formal verification of the $S Q R$-algorithm}

In Figure 6, one can see a flowchart of (a little bit modified) the algorithm of the function SQR from Figure 5. Let us refer to the algorithm as $S Q R$ in the sequel. Having specified the algorithm in the same way as the function, we need to prove the following "relaxation" of the second triple in (3):

$$
[Y, E \in \mathbb{R} \& Y \geq 0 \& E>0] S Q R[|x-\sqrt{Y}| \leq E]
$$

To prove this assertion, let us consider three disjoint cases for the range of the initial value of the variable $Y: 0 \leq Y<1, Y=1$ and $Y>1$ :

$$
\begin{gathered}
{[Y, E \in \mathbb{R} \& 0 \leq Y<1 \& E>0] S Q R[|x-\sqrt{Y}| \leq E]} \\
{[Y, E \in \mathbb{R} \& Y=1 \& E>0] S Q R[|x-\sqrt{Y}| \leq E]}
\end{gathered}
$$




$$
[Y, E \in \mathbb{R} \& Y>1 \& E>0] S Q R[|x-\sqrt{Y}| \leq E] .
$$

Since the second case (6) is trivial, and two other cases, (5) and (7), are very similar, let us consider the last one only, i.e. the assertion (7).

Partial Correctness. Let us employ the Floyd method [2] for a pen-andpaper proof of partial correctness. Let us select the control points 1, 2, and 3 as depicted in Figure 6 to cut the flowchart into three loop-free paths:

path (1..2) from the starting point 1 to point 2;

path $(\mathbf{2 + 3})$ from point 2 to the final point 3 via the positive branch after choice;

path (2-2) from point 2 to the same point 2 via the negative branch after choice.

Let us consider all these paths one by one using the following annotations for the control points:

1. $Y>1 \& E>0$ (i.e. the pre-condition);

2. $Y>1 \& E>0 \& \sqrt{Y}<X \leq Y$ (the loop invariant);

3. $|x-\sqrt{Y}| \leq E$ (i.e. the post-condition).

The first path (1..2) is easy to verify:

$$
\frac{(Y>1 \& E>0) \rightarrow(Y>1 \& E>0 \& \sqrt{Y}<Y \leq Y)}{\{Y>1 \& E>0\} X:=Y\{Y>1 \& E>0 \& \sqrt{Y}<X \leq Y\}} .
$$

The second path $(2+3)$ is not so easy. Let us introduce a test program construct $\phi$ ? as a short-hand for if $\phi$ then stop else abort. Then verification of the path (after some simplification) is as follows:

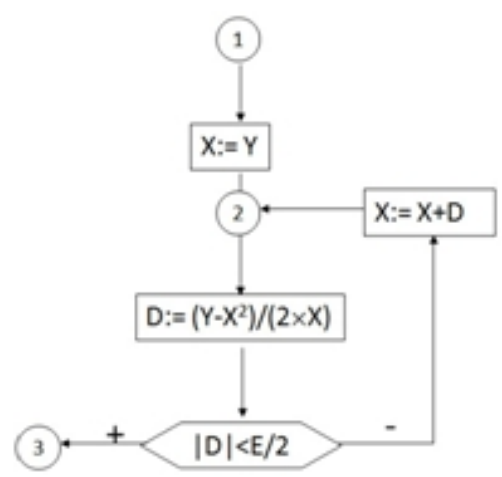

Figure 6. A flowchart of the algorithm $S Q R$ implemented by the function SQR 


$$
\begin{gathered}
\left(Y>1 \& E>0 \& \sqrt{Y}<X \leq Y \&\left|\frac{Y-X^{2}}{2 X}\right|<E / 2\right) \rightarrow|X-\sqrt{Y}|<E \\
\{Y>1 \& E>0 \& \sqrt{Y}<X \leq Y\} D:=\frac{Y-X^{2}}{2 X}\{|D|<E / 2 \rightarrow|X-\sqrt{Y}|<E\} \\
\{Y>1 \& E>0 \& \sqrt{Y}<X \leq Y\} D:=\frac{Y-X^{2}}{2 X} ;|D|<E / 2 ?\{|X-\sqrt{Y}|<E\}
\end{gathered}
$$

The premise

$$
\left(Y>1 \& E>0 \& \sqrt{Y}<X \leq Y \&\left|\frac{Y-X^{2}}{2 X}\right|<E / 2\right) \rightarrow|X-\sqrt{Y}|<E
$$

is valid since in this case we have

$$
|X-\sqrt{Y}|=\left(\frac{|X-\sqrt{Y}|(X+\sqrt{Y})}{2 X}\right) \times\left(\frac{2 X}{X+\sqrt{Y}}\right)<\frac{E}{2} \times \frac{2}{1+\frac{\sqrt{Y}}{X}}<E .
$$

The proof (also after some simplification) of the third path $(2-2)$ is as follows:

$$
\begin{aligned}
\left(Y>1 \& E>0 \& \sqrt{Y}<X \leq Y \&\left|\frac{Y-X^{2}}{2 X}\right| \geq E / 2\right) \rightarrow\left(Y>1 \& E>0 \& \sqrt{Y}<\frac{Y+X^{2}}{2 X} \leq Y\right) \\
\{Y>1 \& E>0 \& \sqrt{Y}<X \leq Y\} D:=\frac{Y-X^{2}}{2 X} \\
\{|D| \geq E / 2 \rightarrow(Y>1 \& E>0 \& \sqrt{Y}<X+D \leq Y)\}
\end{aligned}
$$

$$
\begin{aligned}
\{Y>1 \& E>0 \& \sqrt{Y}<X \leq Y\} D:=\frac{Y-X^{2}}{2 X} ; \\
|D| \geq E / 2 ? ; X:=X+D\{Y>1 \& E>0 \& \sqrt{Y}<X \leq Y\}
\end{aligned}
$$

A hint to prove the premise of this derivation: use the fact that $D<0$ and that the geometric mean is not greater than the arithmetic one $\sqrt{Y}<\frac{Y+X^{2}}{2 X}$.

Termination. Let us observe that the loop invariant $Y>1 \& E>$ $0 \& \sqrt{Y}<X \leq Y$ implies that every loop iteration reduces the absolute value of $D$ by half at least.

Let us fix some $y>1$ as the initial value of the variable $Y, \varepsilon>0$ as the initial value of the variable $E$, let $x_{1}, x_{2}, \ldots x_{n}, x_{(n+1)}, \ldots$ be the values of the variable $X$ immediately before $1^{\text {st }}, 2^{\text {nd }}, \ldots n$-th, $(n+1)$-th, etc., iteration of the loop for this fixed initial value $y$ of $Y$, and let $d_{1}, d_{2}, \ldots d_{n}$, $d_{(n+1)}, \ldots$ be the values of the variable $D$ immediately after $1^{\text {st }}, 2^{\text {nd }}, \ldots$ $n$-th, $(n+1)$-th, etc., iteration of the loop (also for the same fixed initial value $y$ of $Y)$. In particular, $x_{1}=y$ and $d_{n}=\frac{y-x_{n}^{2}}{2 x_{n}}, x_{(n+1)}=x_{n}+d_{n}$ for all n.0.

Let us express $d_{(n+1)}$ in terms of $d_{n}$ :

$$
\begin{aligned}
d_{(n+1)}=\frac{y-x_{(n+1)}^{2}}{2 x_{(n+1)}}=\frac{y-\left(x_{n}+d_{n}\right)^{2}}{2\left(x_{n}+d_{n}\right)}=\frac{y-\left(\frac{y+x_{n}^{2}}{2 x_{n}}\right)^{2}}{2 \frac{y+x_{n}^{2}}{2 x_{n}}} & = \\
& =-\frac{\left(y-x_{n}^{2}\right)^{2} x_{n}}{4 x_{n}^{2}\left(y+x_{n}^{2}\right)}=-\frac{d_{n}^{2} x_{n}}{y+x_{n}^{2}}=-\frac{d_{n}^{2}}{2 x_{(n+1)}} .
\end{aligned}
$$


Note that all values $d_{1}, d_{2}, \ldots d_{n}, d_{(n+1)}, \ldots$ are negative due to loop invariant. Hence

$$
\frac{\left|d_{(n+1)}\right|}{\left|d_{n}\right|}=\frac{d_{(n+1)}}{d_{n}}=-\frac{d_{n}}{2 x_{(n+1)}}=\frac{1}{2} \times \frac{x_{n}^{2}-y}{x_{n}^{2}+y}<\frac{1}{2} .
$$

It implies $\left|d_{(n+1)}\right|<\frac{y}{2^{n}}$, i.e. the algorithm terminates after (at most) $\log _{2} \frac{y}{\varepsilon}$ iterations of the loop.

\section{Concluding remarks}

Let us start these concluding remarks with a quotation from the abstract of paper [12], because it correlates with our paper very well:

Current critical systems commonly use a lot of floating-point computations, and thus the testing or static analysis of programs containing floating-point operators has become a priority. However, correctly defining the semantics of common implementations of floating-point is tricky, because semantics may change with many factors beyond source-code level, such as choices made by compilers. We here give concrete examples of problems that can appear and solutions to implement in analysis software.

The major difference between [12] and the present paper is the concern: the cited paper addresses problems with the floating point value representation and arithmetics, while the present paper considers the problems with the standard functions specification and implementation.

It is worth to remark that a need for better specification and validation of standard functions is recognized (in principle) by industrial and academic professional community, as well as the problem of conformance of their implementation with the specification $[3,10,11,13,14]$.

Paper [3] addresses the formal verification of some low-level mathematical software for the Intel Itanium architecture; in particular, it presents the details of verification of a square root algorithm with the aid of HOL Light theorem prover. The next two papers $[10,11]$ address formal specification and testing of standard mathematical functions. The last two cited papers $[13,14]$ present formal specification and verification of some standard memory management and input-output functions.

A very serious obstacle for the formal verification of standard mathematical functions is a need for the axiomatization of the floating point arithmetic $[1,25]$. The interval analysis approach and formalization of interval arithmetic may help us to tackle the problem for functions like sqrt but not for functions like rand for which we need to develop and employ some special methods for probabilistic programs $[4,16]$. 
Unfortunately, the problem (or a pitfall) of poorly specified and verified standard functions and libraries is still poorly understood by freshmen and inexperienced developers. Better education, specification and verification are needed to solve the problem (and avoid the catch of poor libraries).

Remark. A preliminary very short conference version of this position paper has been published in [15].

Acknowledgement. Many thanks for cooperation and readiness for help to all staff of Information Technologies Dept of IIS and especially to language editor Anna Shelukhina!

\section{References}

[1] Ayad A., Marché C. Multi-prover verification of floating-point programs // Lect. Notes in Artificial Intelligence. - 2010. - Vol. 6173. - P.127-141.

[2] Gries D. The Science of Programming. - Springer-Verlag, 1981.

[3] Harrison J. Formal verification of square root algorithms // Formal Methods in System Design. - 2003. - Vol. 22, No. 2. - P.143-153.

[4] Den Hartog J.I., de Vink E.P. Verifying probabilistic programs using a Hoare like logic // Internat. J. of Foundations of Computer Science. - 2002. - Vol. 13, No. 3. - P.315-340.

[5] Hoare C.A.R. The verifying compiler: a grand challenge for computing research // Lect. Notes Comput. Sci. - 2003. - Vol. 2890. - P. 1-12.

[6] Gutowski M.W. Power and beauty of interval methods. arXiv:physics/0302034 [physics.data-an]. urlhttp://arxiv.org/pdf/physics/0302034.pdf (visited January 19, 2016).

[7] Exercise $\sharp 8 / /$ Programming in C: A Complete Introduction to the C Programming Language (3rd Edition) / S.G. Kochan. - Sam's Publishing, 2005. - P. 162-163.

[8] Functions Calling Functions Calling // Programming in C: A Complete Introduction to the C Programming Language (3rd Edition) / S.G. Kochan. Sam's Publishing, 2005. - P. 131-137.

[9] Kolmagorov A.N., Fomin S.V. Elements of Funcions Theory and Functional Analysis (4th ed.). - Nauka Publishers, 1976 (In Russian).

[10] Kuliamin V. Standardization and testing of mathematical functions // Programming and Computer Software. 2007. - Vol. 33, No. 3. - P. 154-173.

[11] Kuliamin V.V. Standardization and testing of mathematical functions in floating point numbers // Lect. Notes Comput. Sci. - 2010. - Vol. 5947. - P. 257268. 
[12] Monniaux D. The pitfalls of verifying floating-point computations // ACM Trans. on Programming Languages and Systems. - 2008. - Vol. 30, No. 3. P. 1-41.

[13] Promsky A.V. C Program Verification: Verification Condition Explanation and Standard Library // Automatic Control and Computer Sciences. - 2012. - Vol. 46, No. 7. - P. 394-401.

[14] Promsky A.V. Experiments on self-applicability in the C-light verification system // Bulletin NCC. Series: Computer Science. - 2013. - Iss. 35. - P. 85-99.

[15] Shilov N.V. A Need To specify and verify standard functions // Proc. Internat. Conf. Tools \& Methods of Program Analysis (TMPA-2015, November 12-14, 2015). - Saint-Petersburg State Polytechnical University. - P.119-122.

[16] Rand R., Zdancewic S. VPHL: a verified partial-correctness logic for probabilistic programs // Electronic Notes in Theor. Comput. Sci. - 2015. - Vol. 319. - P.351-367.

[17] Pi. Memorizing digits. - https://en.wikipedia.org/wiki/Pi\#Memorizing_ digits (visited January 19, 2016).

[18] $\mathrm{Pi}$ Function. - https://support.office.com/en-us/article/ PI-function-264199d0-a3ba-46b8-975a-c4a04608989b (visited January 19, 2016).

[19] Stanislaw Jerzy Lec Quotes. - http://www.azquotes.com/author/ 8631-Stanislaw_Jerzy_Lec (visited January 19, 2016).

[20] C reference. - Rand.http://en.cppreference.com/w/c/numeric/random/ rand (visited January 19, 2016).

[21] How to make a program that solves the quadratic formula. - http://www . youtube. $\mathrm{com} /$ watch?v=15NbFrBUdu0 (visited January 19, 2016).

[22] Write a $\mathrm{C}++$ program that solves quadratic equation to find its roots. - http: //www.cplusplus .com/forum/general/36313/ (visited January 19, 2016).

[23] C refernce. Sqrt, sqrtf, sqrtl. - http://en.cppreference.com/w/c/numeric/ math/sqrt (visited January 19, 2016).

[24] ISO/IEC/IEEE 60559:2011. - Information technology - Microprocessor Systems - Floating-Point arithmetic http://www.iso.org/iso/iso_catalogue/ catalogue_tc/catalogue_detail.htm?csnumber=57469 (visited January 19, 2016).

[25] Hisseo. - http://hisseo.saclay.inria.fr/index.html (visited January 19, 2016). 
